\title{
A novel homozygous variant of GPR98 causes usher syndrome type IIC in a consanguineous Chinese family by next generation sequencing
}

Chunli Wei ${ }^{1,2}$, Lisha Yang ${ }^{2}$, Jingliang Cheng ${ }^{2}$, Saber Imani ${ }^{2,3}$, Shangyi Fu ${ }^{4,5}$, Hongbin Lv ${ }^{6}$, Yumei Li ${ }^{5}$, Rui Chen ${ }^{5}$, Elaine Lai-Han Leung ${ }^{1,7,8^{*}}$ and Junjiang $\mathrm{Fu}^{1,2^{*}}$ (D)

\begin{abstract}
Background: Usher syndrome (USH) is a common heterogeneous retinopathy and a hearing loss (HL) syndrome. However, the gene causing Usher syndrome type IIC (USH2C) in a consanguineous Chinese pedigree is unknown.

Methods: We performed targeted next-generation sequencing analysis and Sanger sequencing to explore the GPR98 mutations in a USH2C pedigree that included a 32-year-old male patient from a consanguineous marriage family. Western blot verified the nonsense mutation.

Results: To identify disease-causing gene variants in a consanguineous Chinese pedigree with USH2C, DNA from proband was analyzed using targeted next generation sequencing (NGS). The patient was clinically documented as a possible USH2 by a comprehensive auditory and ophthalmology evaluation. We succeeded in identifying the deleterious, novel, and homologous variant, c.6912dupG (p.Leu2305Valfs*4), in the GPR98 gene (NM_032119.3) that contributes to the progression of USH2C. Variant detected by targeted NGS was then confirmed and cosegregation was conducted by direct Sanger sequencing. Western blot verified losing almost two-thirds of its amino acid residues, including partial Calx-beta, whole EPTP and 7TM-GPCRs at the C-terminus of GPR98. Furthermore, our results highlighted that this p.Leu2305Valfs*4 variant is most likely pathogenic due to a large deletion at the seven-transmembrane G protein-coupled receptors (7TM-GPCRs) domain in GPR98 protein, leading to significantly decreased functionality and complex stability.

Conclusions: These findings characterized the novel disease causativeness variant in GPR98 and broaden mutation spectrums, which could predict the pathogenic progression of patient with USH2C, guide diagnosis and treatment of this disease; and provide genetic counseling and family planning for consanguineous marriage pedigree in developing countries, including China.
\end{abstract}

Keywords: Usher syndrome type IIC, GPR98, Nonsense mutation, Molecular diagnosis, Next generation sequencing, Consanguineous marriage

\footnotetext{
* Correspondence: Ihleung@must.edu.mo; fujunjiang@hotmail.com

${ }^{1}$ State Key Laboratory of Quality Research in Chinese Medicine, Macau

Institute For Applied Research in Medicine and Health, Macau University of

Science and Technology, Taipa, Macao, Special Administrative Region of

China

Full list of author information is available at the end of the article
}

C The Author(s). 2018 Open Access This article is distributed under the terms of the Creative Commons Attribution 4.0 International License (http://creativecommons.org/licenses/by/4.0/), which permits unrestricted use, distribution, and reproduction in any medium, provided you give appropriate credit to the original author(s) and the source, provide a link to the Creative Commons license, and indicate if changes were made. The Creative Commons Public Domain Dedication waiver (http://creativecommons.org/publicdomain/zero/1.0/) applies to the data made available in this article, unless otherwise stated. 


\section{Background}

Usher syndrome (USH) is a common heterogeneous retinopathy and a hearing loss (HL) syndrome; the disease is also known as Usher-Hallgren syndrome, Hallgren syndrome, or retinitis pigmentosa (RP)-dysacusis syndrome $[1,2]$. It has high prevalence, often estimated to be roughly 1 in 6000 individuals and 1 in 6 people with $\mathrm{RP}$, covering nearly $50 \%$ of all deaf-blindness cases $[3,4]$. USH is mostly inherited in an autosomal recessive pattern and its clinical subtypes can be determined by the presence of arreflexia of sensorineural HL: USH1, USH2, and USH3 $[2,5]$.

Pathogenically, Usher syndrome is mapped by at least eleven $U S H$ genes which contribute to inheritance in USH1: MYO7A, USH1C, CDH23, PCDH15, USH1G, USH2A, GPR98, DFNB31, CLRN1, and PDZD7 [6-11]. Usher syndrome type II is the most common form of USH syndrome, leading to severe RP in the second decade of life along with congenital, moderate-to-severe HL. To date, USH2 loci are mutagenetically heterogeneous in three different genes: USH2A (USH2A, OMIM 276901) [12], GPR98 (USH2C, OMIM 605472) [8, 13], and DFNB31 (USH2D, OMIM 611383) [14]. PDZD7 is a modifier gene found in USH2 patients [15]. To our knowledge, USH2A is the most prevalent mutated gene in USH2 patients, consisting of an estimated $80 \%$ of all patients inflicted with USH2, whereas GPR98 mutations account for a small but significant number of inflicted patients [16].

The human G protein-coupled receptor 98 (GPR98) gene (NM_032119.3), located at chromosome 5q14.3, is also aliased as FEB4, USH2B, USH2C, VLGR1, VLGR1b, ADGRV1 and MASS1. The protein, encoded by GPR98 gene, is a part of an Usher interactome as G proteincoupled receptors (GPCRs). Functionally, GPCRs, as a seven-transmembrane domain receptors (7TM receptors) (7TM-GPCRs), are integral membrane proteins that possess seven membrane-spanning domains or transmembrane helices [17]. In USH2C patients, GPCRs are critical for the proper hair cell development and maintenance of photoreceptors structures, in which it may allow connecting cilium to anchor the inner segment of the photoreceptors [18]. As a seventransmembrane receptor in the retinal cells, the extracellular loops of GPCRs also contain two highly conserved cysteine residues that form disulfide bonds to stabilize the receptor structure $[19,20]$. Even with emerging evidence, GPCR's function in the USH2 disease is not well described yet.

Although USH2C has been studied in regard to mutational screening, characterization of different variants in the whole region of GPR98 gene, is still unknown. Clinically and genetically, this is a necessary issue to be addressed as the USH2C patients who desperately need genetic counseling have no definitive answer $[15,19]$.
Next generation sequencing (NGS) is one of the newest, most cost-effective molecular analysis technique mainly used to screen pathogenic variants in inherited heterogeneous disorders [21-23]. In this study, we aimed to categorize pathogenic and disease-causing variants by targeted NGS technologies-based mutation screening in a Chinese USH2C pedigree with consanguineous marriage. Our results successfully found a deleterious, novel, and pathogenic mutation c.6912dupG (p.Leu2305Valfs"4), in the GPR98 gene. This less commonly mutated gene could possibly predict the progression of USH2C, contributing to the progression of causativeness and susceptibility in USH2C patient. This study provides detailed information that will help find the genetic and clinical data to support the contention that the autosomal recessive mutation in GPR98 is most likely pathogenic by damaging the GPR98 protein structure and protein/protein interaction.

\section{Methods \\ Patient recruitments}

This research was approved by the Ethical Committees of the Southwest Medical University. All written informed consents and procedures adhered to the tenets of the Declaration of Helsinki (1983 Revision). A written informed consent to genetic testing was obtained from all enrolled subjects according to the recommendations of the local ethics committee of Southwest Medical University before inclusion into the study. A pedigree for a consanguineous marriage, five-generations, elevenChinese members including one patient proband with both hearing loss and vision defect, was enrolled (Fig. 1, pedigree IV: 1, arrow, molecular No. M203). A complete historic interview enabled us to draw an extended pedigree from the family and to confirm the inheritance pattern. The proband has been clinically evaluated according to the criteria recommended by the USH Consortium [20]. A comprehensive auditory and ophthalmology evaluation was performed upon the proband, including the followings: otoscope examination, tympanometry, best corrected visual acuity (BCVA) measurements, slit-lamp biomicroscopy and color vision, fundus photography, visual field tests. The retinal phenotypes, thickness, and structure change of proband were examined by full field electroretinograms and optical coherence tomography instruments (OCT, Carl Zeiss, Germany), according to the standard protocol. Audiograms were measured by pure-tone audiometry at different frequencies of $0.25,0.5,1,2,4$, and $8 \mathrm{kHz}$ in the proband.

\section{DNA sampling}

Whole blood samples from all members of the M203 pedigree were collected (Fig. 1). Human genomic DNAs 




Fig. 1 The pedigree for USH2C in Chinese with consanguineous marriage. Family numbers and disease-causing variant is noted above pedigree. Normal individuals are shown as clear circles (females) and squares (males), whereas affected individual is shown as a filled symbol. III:1 and III:2 are consanguineous marriage with symbol " $=$ ". The patient above the arrow indicates the proband (IV:1). The arrow indicates the patient by next generation sequencing with G duplication mutation of GPR98 gene NM_032119.3: c.6912dupG: p.Leu2305Valfs*4. " $M$ " indicates the mutant allele of GPR98, whereas " $N$ " indicates normal allele without mutation

(gDNA) from peripheral leukocytes were extracted using the previously described phenol/chloroform method [24, 25]. The concentration of the extracted gDNAs was measured by a NanoDrop2000 spectrophotometer (Thermo Scientific, Wilmington, DE, USA). DNAs from 100 ethnically matched Chinese individuals without any hearing and retinal diseases were recruited as normal controls.

\section{Targeted NGS and data analysis}

Sequencing analyses were performed as described using the capture Agilent probes that was used in previously published studies [22, 26, 27]. The design of targeted capture panels with 195 genes has been described in previous literature, according to the Illumina paired-end libraries [22, 26, 28]. Variant filtering and homozygosity mapping were performed using a paired-end sequencing Illumina, which was aligned to the human hg19 reference genome using Burrows-Wheeler Aligner version 0. 6.1 and available public online UCSC database (http:// genome.ucsc.edu/) [29], as previously described in detail $[22,26]$. The data from targeted NGS were analyzed as described [21, 27, 30]. The functional classification of proteins via subfamily domain architectures for GPR98 was performed through an online system (https://www. ncbi.nlm.nih.gov/Structure/cdd/wrpsb.cgi) [30].

\section{Variant verification and segregation analysis}

The locus-specific primers for variant verification and segregation analysis were designed using online Primer3 program (website: http://bioinfo.ut.ee/primer3-0.4.0/) (left primer 5'-GGGACACTCGGCAATGTTAC-3'; right primer 5' - CCAAAATGTTGAACAATGCAA -3'). The mutant position should locate at least 50 bps away from the 3 '-end of both left and right primers. A product with 422 bps was amplified by PCR using gDNA as the template [25]. Sanger sequencing was conducted to validate the suspected pathogenic variants and segregation analysis using 3500DX ABI Genetic Analyzer (Foster City, USA).

\section{Western blot analysis}

One $\mathrm{ml}$ of blood from the affected proband with mutant homozygous type (pedigree IV:1, Fig. 1); one $\mathrm{ml}$ of blood from the mutant heterozygous type (M204) (pedigree III: 1, Fig. 1); and one $\mathrm{ml}$ of blood from the wild type individual in this Chinese family (pedigree IV:2, Fig. 1) were collected, respectively, to isolate the leukomonocytes from the red cells using lymphocyte separation medium (TBD, China), and then lysed using the EBC lysis buffer [31, 32]. The concentration of the proteins was determined with protein quantification kit-Rapid (Sigma, USA). Then, lysates were separated onto a 7\% SDS-polyacrylamide gel and transferred to a PVDF membrane (Millipore, Corporation, Billerica, MA, USA). The membrane was blocked with $5 \%$ milk without fat in $1 \times$ TBST buffer $(1 \times$ TBS plus $0.05 \%$ Tween-20) for $2 \mathrm{~h}$ at room temperature. Primary GPR98 antibody (Cat \#: abs139741a, Absin Bioscience Co. , Ltd., Shanghai, China) with 1:1000 dilution and tubulin antibody (Cat \#: T0198, Sigma, USA) with 1:2500 dilution were incubated respectively at $4{ }^{\circ} \mathrm{C}$ for $13 \mathrm{~h}$ with gentle shaking. After the membranes were washed three times with $1 \times$ TBST buffer for $15 \mathrm{~min}$ per wash, the secondary antibodies tagged with horseradish peroxidase (HRP) (1:2000 dilution) were added to the membranes and incubated at room temperature for $2 \mathrm{~h}$ with gentle shaking. After another three washes with $1 \times$ TBST buffer for $10 \mathrm{~min}$ per wash, protein bands were recorded by the digital imaging system (Universal Hood II, Bio-Rad Lab, Italy) [32].

\section{Results}

\section{Phenotype for proband with usher syndrome}

The pedigree with consanguineous marriage was recruited from Department of Ophthalmology in Southwest Medical University (Fig. 1) when the proband was 32-year-old. The father of proband claimed his son was found to have hearing defects when he was one and half year old during otitis media therapy at the local county hospital, and had a gradual decrease of vision, which was revealed when he turned 20-year-old. Pure tone audiometry testing presented 
prelingual deafness with bilateral slightly downsloping and showed a moderate-to-severe sensorineural hearing loss across all frequencies (Fig. 2a). The retinal phenotypes of the proband were compared to the normal control (Fig. 2b-e). The presenting symptoms of proband were shown as decreased central vision and visual acuity, and prominent presence of fundus flecks in the posterior pole of the retina. The FA (fluorescein angiogram) results (pedigree IV:1, Fig. 1) showed a "salt and pepper" pigment mottling pattern; severe RPE atrophic changes and the transparency of the macula; and fundus abnormalities (Fig. 2b-c). Optical coherence tomography (OCT) showed that marked thinning and disruption of the photoreceptor layer, choroid and the retinitis pigment epithelium (Fig. 2e). There was no history of imbalance gait or vertigo. Thus, it was determined that the patient had likely presence of USH2 (Fig. 1). Family history has shown no other members with hearing losses or visual problems.

\section{Mutation identification for the GPR98 gene}

The proband was analyzed using target capture panel sequencing. This panel covers coding exons and flanking splicing junctions for 195 known retinal disease genes, including GPR98 [22, 28]. High quality next-generation sequencing data were obtained. The NGS analysis in the proband identified homozygous variant c.6912dupG for the GPR98 gene (NM_032119.3). We successfully identified the duplication mutation was c. 6912dupG in exon 31 , resulting in a missense change of Leucine at position 2305 to Valine and frameshift mutation with termination codon at position 2310 on GPR98 protein (p.Leu2305Valfs*4). After checking this variant on the exome variant server and ExAC databases showed this variation is novel. This mutation has not been reported in single nucleotide polymorphisms, and is perfectly co-segregated with the USH2 phenotype shown by Sanger sequencing. The c.6912dupG variant was not found in 100 unrelated, ethnically matched normal controls. The representative results of the Sanger sequencing of this variant are shown in Fig. 3. As we estimated, the consanguineous proband's parents (Fig. 3a\&b, pedigree: III: 1\&2) were heterozygous for c.6912dupG. The proband was homozygous for the mutation of GPR98 (Fig. 3c), whereas his wife (pedigree IV: 2, Fig. 1) was homozygous for the wild type allele of GPR98 (Fig. 3d). The proband wife genotype is not relevant since there is no consanguinity. This confirmation results showed comprehensive cosegregation in this pedigree family association furthering population studies and pinpointing the roles in USH2C pathogenesis.

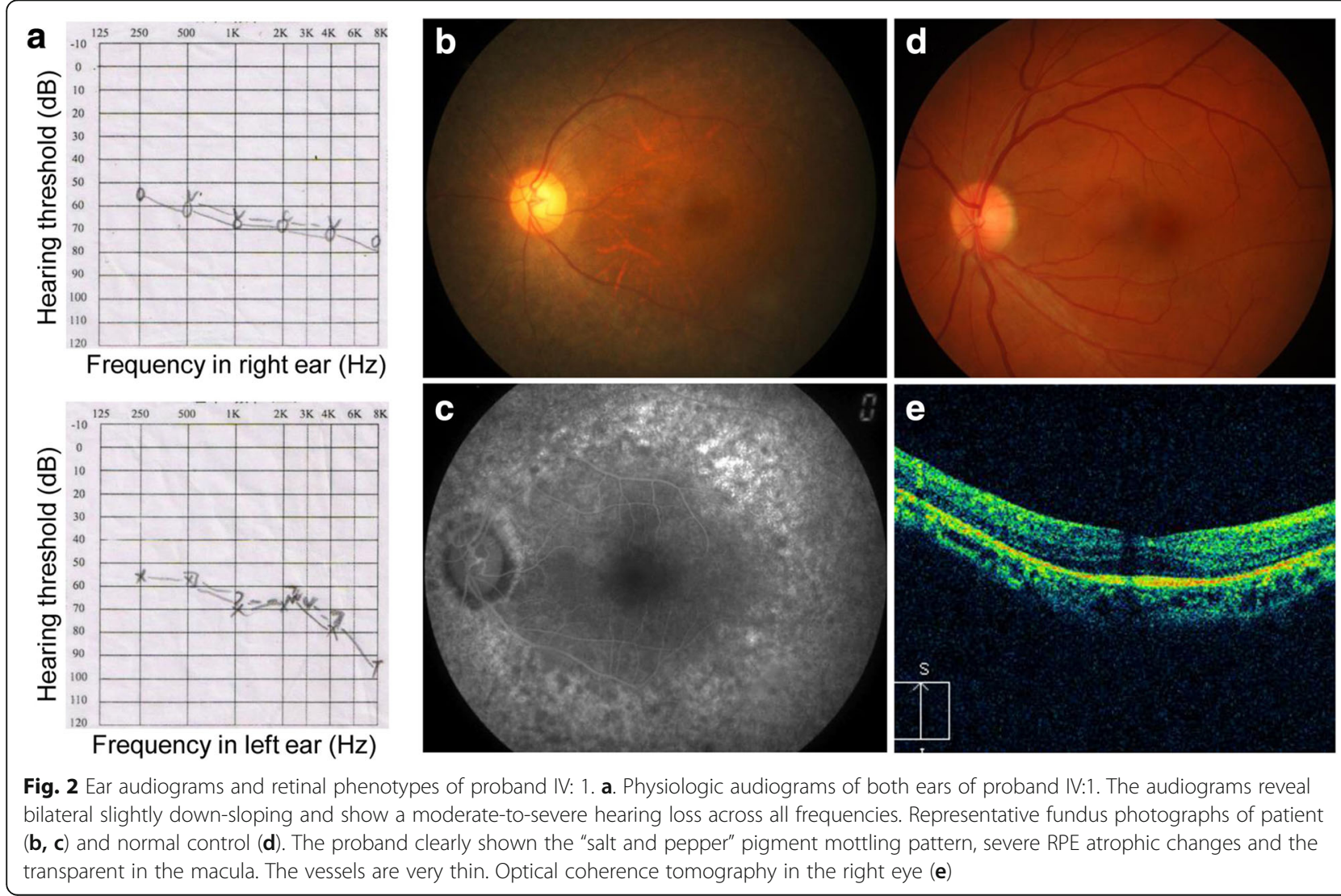




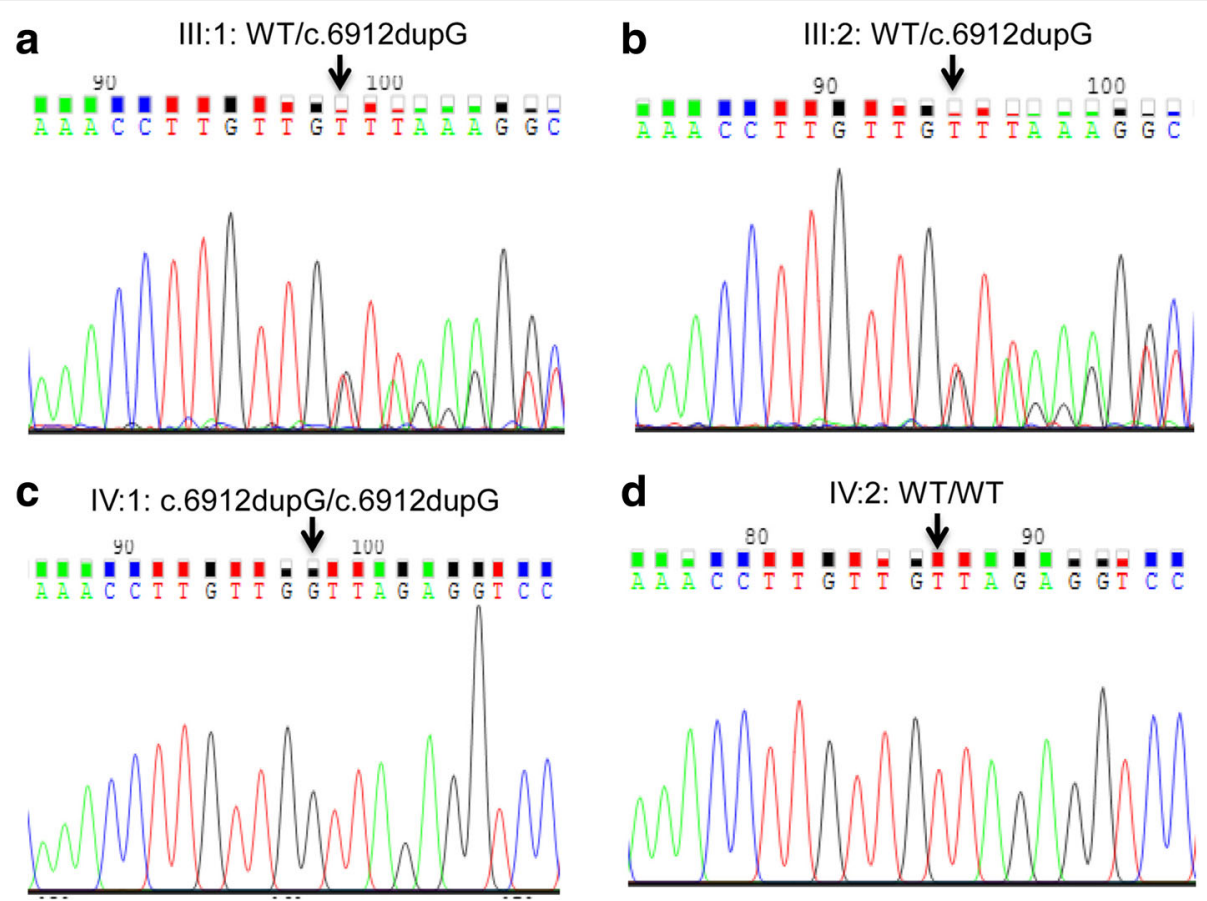

Fig. 3 Validation and segregation analysis by Sanger sequencing. a, b, c and $\mathbf{d}$ indicate the sequencing results in III: 1 (M204, heterozygous type), III: 2 (heterozygous type), IV: 1 (M203, mutant homozygous type) and IV:2 (wild type, normal control: no eye and ear disease history in her family), respectively. The arrows indicate the duplication at the nucleotide position for GPR98 gene NM_032119.3: c.6912dupG: p.Leu2305Valfs*4

\section{Change of GPR98 protein}

We recognized a single nucleotide homozygous duplication (c.6912dupG) of GPR98 gene in this USH2C family, leading to a missense change of Leucine at position 2305 to Valine and a frameshift mutation in the reading frame at amino acid position 2305 and another 4 incorrect amino acids (RGPG) after codon 2305, followed by premature termination at codon 2310 (p.Leu2305Valfs*4), with a total of 2309 amino acids of mutant GPR98 protein (Fig. 4). Conserved domains analysis for GPR98 protein amino acid residues of the wild type and the mutant c.6912dupG: p.Leu2305Valfs"4 revealed that the wild type protein contains domains Laminin-G-3, EPTP, Calx-beta (green box), and 7TM-GPCRs, whereas the mutant protein only contains domains Laminin-G-3, and Calx-beta, losing almost two-thirds of its amino acid residues, including partial Calx-beta, whole EPTP and 7TM-GPCRs at the C-terminus of GPR98, which is considered as the cause of the disease. Notable, EPTP domain is a common protein interaction domain links identified epilepsy gene Epitempin.

To verify the predicted change in GPR98 protein, the GPR98 in proband (pedigree IV:1, Fig. 1) was compared to the heterozygous type (pedigree III:1, Fig. 1), the wild type, and the normal control (pedigree IV:2, Fig. 1), respectively, by western blot analysis. The results are shown in Fig. 5a; It is worthy to note that a smaller band (GPR98-S; $170 \mathrm{KD}$ ) was presented only in mutant homozygous type of USH2C patient (pedigree IV:1, Fig. 5a, lane "IV1") and a larger (GPR98-L; $690 \mathrm{KD}$ ) band was presented only in wild type (normal control) (pedigree IV:2, Fig. 5a, lane "WT") in GPR98

\begin{tabular}{|l} 
GPR98 \\
G.L2305fs \\
Fig. 4 The conserved domains analysis for GPR98 amino acid residues wild type and its mutant protein c.6912dupG: p.Leu2305Valf**4. The conserved \\
domains analysis was performed through the online system (https:/www.ncbi.nlm.nih.gov/Structure/cdd/wrpsb.cgi). Wild type protein contains \\
domains Laminin-G-3, EPTP, Calx-beta (green box), and 7TM-GPCRs, whereas mutant protein only contains domains Laminin-G-3, and Calx-beta, which \\
loses almost two-thirds of amino acid residues including partial Calx-beta, and whole EPTP and 7TM-GPCRs. Laminin-G-3: Laminin G domain; 7TM- \\
GPCRs: seven-transmembrane (7TM) G protein-coupled receptors (GPCRs); GPS: GPCR proteolysis site (motif). "p.L2305 fs" indicates "p.Leu2305Valfs*4"
\end{tabular}




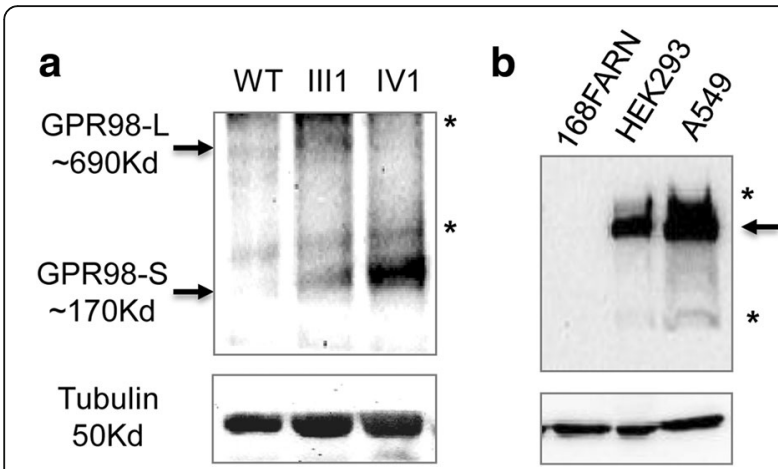

Fig. 5 Western blot results of GPR98. a. Western blot results for samples III: 1 (M204), IV: 1 (M203, mutant homozygous type) and IV: 2 (wild type, normal control), respectively. b. Western blot results for cancer cell lines. The arrows indicate the large size (GPR98-L) and/or small size (GPR98-S) forms of GPR98 with p.Leu2305Valfs*4. "**" in the image indicates non-specific bands, respectively

protein, respectively. This smaller band is the probable, truncated GPR98 protein due to the frameshift mutation (p.Leu2305Valfs*4). In the heterozygous type of USH2C carrier III: 1, we identified both a smaller band (GPR98-S; 170 KD) and a larger band (GPR98-L; 690 KD) (Fig. 5a, lane "III1"). We also noticed that the signals of smaller band GPR98-S in proband (IV:1) with homozygous mutation are stronger than that of carrier (III:1) with heterozygous mutation due to copy number difference of mutant GPR98 gene. To make sure whether SDS-PAGE of 7\% acrylamide can used to detect a large protein of 700 KD successfully, different cancer cell lines were used for WB, which do show specific band with GPR98 (Fig. 5b and data not shown). These findings suggest that the c.6912dupG: p.Leu2305Valfs*4 variant in the USH2C patient could affect the GPR98 protein function, by causing a premature frameshift in the structure.

\section{Discussion}

By identifying GPR98 mutation, the current study retrospectively performed targeted NGS on USH Chinese family with consanguineous marriage to identify a disease-causing variant responsible for the USH2C syndrome (Usher Syndromes type IIC) which thereafter characterized at the molecular level. USH2C is usually characterized by moderate-to-severe hearing loss, latter onset of retinal degeneration and normal vestibular function [2, 8, 33]. For the first time, our findings introduced this family with a novel c.6912dupG: p.Leu2305Valfs*4 GPR98 frameshift mutations, which lead to protein truncation. These findings highlighted that this novel variant in the GPR98 is likely the deleterious variant, thereby expanding the GPR98 mutation spectrums for USH2C syndrome.
Clinical subtyping of USH hinders the critical therapy step, which allows early molecular diagnosis of patient's families through genotype-phenotype descriptions and characterization of any new disease-causing gene variants in the molecular laboratory [16, 20]. Following this approach, USH mutation genes were documented and clinically used for subtypes of Usher or other retinal diseases. GPR98 mutations account for a small portion for mutation that causes USH2C. Till now, ten deleterious mutations, including small insertions, point mutations, deletions, and splicing alterations were identified in GPR98 [34]. This patient is successfully characterized as USH2C syndrome by combination of GPR98 mutation identification, hearing loss observation, the retinal pigment epithelium abnormality and rod-cone degeneration [11].

It is well known that NGS is one of the newest, most cost-effective molecular analysis technique mainly used to identify pathogenic variants in inherited heterogeneous disorders [21, 22, 27]. Literature reviews show NGS is a predominantly accurate technique which adds to previous differential imaging techniques that have been able to characterize and map more genetically heterogeneous macular degeneration/dystrophy (MD) disorder, such as RP, STGD-like MD, LCA, and USH $[28,35,36]$. To address this issue, according to NGSbased models, we have succeeded in identifying a new GPR98 disease-causing variant in a Chinese USH2C family (c.6912dupG: p.Leu2305Valfs*4).

The encoded GPR98 protein (NP_115495.3) is a very large protein found in humans, with a size of 6306 amino acids and consisting of 90 exons (NM_032119.3, largest isoform b with predicted 605KD). Structurally, it belongs to the subfamily of the GPCR2 family for Gprotein signaling or large N-terminal family B (LNB) of 7TM receptors (LN-TM7 subfamily) [37]. Specifically, this protein, which has several domains, is detected to be $\sim 690 \mathrm{KD}$ in molecular mass by western blot (Fig. 5), involved in the signaling pathways of GPCRs in the inner ear and retinal cells, and possibly leads to variable ratios of mutant/normal transcripts in these cells $[3,38]$. Its function in the retinal cells guaranteed photoreceptor cell maintenance and visual perception of the retinal [20]. Similarly, the GPR98 protein may localize in specific subcellular compartments in spiral ganglion cells of the cochlea that assist in maintaining stereociliary cohesion [3]. The mutation's gene expression may affect the cochlear stereociliary bundle and cause hearing loss [38, 39].

In last decade, homozygous mutations in the GPR98 gene were reported in a consanguineous Tunisian family [40], Iranian family [8], German family [41], and French family [41], all of which are linked to USH2C syndrome phenotype. These genotype/phenotype correlations are helpful in the verification of GPR98 function in the USH2C syndrome [42]. Most of mutations are located in 
the 7TM-GPCRs functional domain in the GPR98 gene. All predicted premature terminations have led to the dysfunction of the GPR98 complex, suggesting that this protein participates in cell adhesion and signal transduction of sensory retinal cells. In this study, we first time report a novel, large deletion of the 7TM-GPCRs of GPR98 protein in a large consanguineous Chinese USH2C family. Conserved domain analysis for GPR98 protein shows this region of GPR98 is highly conserved and predicts a globular domain that is involved in retinal cell-cell signaling pathways $[20,30]$. The frameshift of deleterious c.6912dupG: p.Leu2305Valfs* 4 variant might distribute protein abnormal location and affect normal protein/protein interactions after deleting the functional domain of GPR98 [43]. USH2A, GPR98, WHRN and PDZD7 interact to assemble a multiprotein complex at the ankle link region of the mechanosensitive stereociliary bundle in hair cells. Defects of any proteins in this complex should cause stereociliary bundle disorganization and deafness. From this analysis, we suggested that the mutated GPR98 domain has drastically decreased protein's functionality and complex stability due to the partial unfolding of the 7TM-GPCRs domain. Hopefully, NGS technique with the molecular simulation trajectory will be guidance in revealing the potential phenotype-genotype correlation, and diagnosis and treatment of this disease [44].

The offspring of consanguineous marriages are at higher risk of genetic disorders. Autosomal recessive disorders carry two copies or alleles of the same GPR98 gene mutation in the consanguineous marriage, one of each inherited from their grandparents. Both parents of an individual with this USH2C are carriers of GPR98 gene, displaying no signs of diseases, and might not be aware that they carry the same mutated gene. However, their children are at a $25 \%$ risk in those autosomal recessive disorder families. In this regard, it is very important to provide genetic counseling and family planning for the consanguineous marriage pedigree in un-developed and developing countries, including Tunisia and China.

\section{Conclusions}

Our findings showed the possibility that the novel homozygous p.Leu2305Valfs"4 frameshift variant of GPR98 is deleterious and disease-causing, according to the NGS-based comprehensive genetic evaluation of a $\mathrm{USH} 2 \mathrm{C}$ variation in a consanguineous marriage Chinese family. These results may help our understanding of the variant that contributes to the susceptibility or causativity of USH progression. Thus, our study indicates that p.Leu2305Valfs*4 variant could be novel and pathogenic, that risks for carriers and their potential offspring must be informed to USH2C patients and their families. Genetic counseling and family plan for consanguineous marriage pedigree is also necessary.

\section{Abbreviations}

FA: Fluorescein angiogram; GPR98: G protein-coupled receptor 98; NGS: Next generation sequencing; OCT: Optical coherence tomography; USH2C: Usher syndrome type $\| C$

\section{Acknowledgements}

We gratefully acknowledge the patient and his family, volunteers for collaborations with us in sampling during of the project.

\section{Funding}

This work was supported in part by the National Natural Science Foundation of China (30371493, 31701087, 81172049, 81672887), the Research

Foundation of the Education Department of Sichuan Province (17ZA0427 17ZB0467), the Science and Technology Innovation Team of Colleges and Universities of Sichuan Province (13TD0032), the Research Foundation of the Science and Technology Department of Luzhou City (2015-S-42(3/4), 2016-S$65(9 / 9))$

\section{Authors' contributions}

$C W, J C, L Y$, and SF conducted experiments; CW and JF prepared the figures; $\mathrm{RC}$ and $\mathrm{YL}$ performed target NGS and analyzed the data; HL recruited samples and performed clinical examination. SI, SF and JF wrote the manuscript; CW, SF and JF revised the manuscript; JF and EL planned and supervised the project. All authors reviewed the manuscript. All authors read and approved the final manuscript.

\section{Ethics approval and consent to participate}

The study was approved by Southwestern Medical University and written informed consent was obtained from all subjects.

\section{Consent for publication}

Written informed consent was obtained from the participants for publication of medical data and images.

\section{Competing interests}

The authors declare that they have no competing interests.

\section{Publisher's Note}

Springer Nature remains neutral with regard to jurisdictional claims in published maps and institutional affiliations.

\section{Author details}

${ }^{1}$ State Key Laboratory of Quality Research in Chinese Medicine, Macau Institute For Applied Research in Medicine and Health, Macau University of Science and Technology, Taipa, Macao, Special Administrative Region of China. ${ }^{2}$ Key Laboratory of Epigenetics and Oncology, Research Center for Preclinical Medicine, Southwest Medical University, Luzhou, Sichuan, China. ${ }^{3}$ Chemical Injuries Research Center, Baqiyatallah University of Medical Sciences, Tehran, Iran. ${ }^{4}$ The Honors College, University of Houston, Houston, TX, USA. ${ }^{5}$ Department of Molecular and Human Genetics, Baylor College of Medicine, Houston, TX 77030, USA. ${ }^{6}$ Department of Ophthalmology, Affiliated Hospital of Southwest Medical University, Luzhou, Sichuan, China. ${ }^{7}$ Guangzhou Institute of Respiratory Disease, State Key Laboratory of Respiratory Disease, The 1st Affiliated Hospital of Guangzhou Medical College, Guangzhou, China. ${ }^{8}$ Respiratoire Medicine Department, Taihe Hospital, Hubei University of Medicine, Hubei, China.

Received: 12 February 2018 Accepted: 1 May 2018

Published online: 11 June 2018

References

1. Keats BJ, Corey DP. The usher syndromes. Am J Med Genet. 1999;89(3): $158-66$.

2. Millan JM, Aller E, Jaijo T, Blanco-Kelly F, Gimenez-Pardo A, Ayuso C. An update on the genetics of usher syndrome. J Ophthalmol. 2011;2011:417217.

3. Boughman JA, Vernon M, Shaver KA. Usher syndrome: definition and estimate of prevalence from two high-risk populations. J Chronic Dis. 1983; 36(8):595-603

4. Espinos C, Millan JM, Beneyto M, Najera C. Epidemiology of usher syndrome in Valencia and Spain. Community genetics. 1998;1(4):223-8. 
5. Williams DS. Usher syndrome: animal models, retinal function of usher proteins, and prospects for gene therapy. Vis Res. 2008;48(3):433-41.

6. Eudy JD, Weston MD, Yao S, Hoover DM, Rehm HL, Ma-Edmonds M, Yan D, Ahmad I, Cheng JJ, Ayuso C, et al. Mutation of a gene encoding a protein with extracellular matrix motifs in usher syndrome type Ila. Science. 1998; 280(5370):1753-7.

7. Pieke-Dahl S, Moller CG, Kelley PM, Astuto LM, Cremers CW, Gorin MB, Kimberling WJ. Genetic heterogeneity of usher syndrome type II: localisation to chromosome 5q. J Med Genet. 2000;37(4):256-62

8. Hilgert N, Kahrizi K, Dieltjens N, Bazazzadegan N, Najmabadi H, Smith RJ, Van Camp G. A large deletion in GPR98 causes type IIC usher syndrome in male and female members of an Iranian family. J Med Genet. 2009;46(4):272-6.

9. Ouyang XM, Yan D, Du LL, Hejtmancik JF, Jacobson SG, Nance WE, Li AR, Angeli S, Kaiser M, Newton V, et al. Characterization of usher syndrome type I gene mutations in an usher syndrome patient population. Hum Genet. 2005;116(4):292-9.

10. Jaijo T, Aller E, Garcia-Garcia G, Aparisi MJ, Bernal S, Avila-Fernandez A, Barragan I, Baiget M, Ayuso C, Antinolo G, et al. Microarray-based mutation analysis of 183 Spanish families with usher syndrome. Invest Ophthalmol Vis Sci. 2010;51(3):1311-7.

11. Le Quesne SP, Saihan Z, Rangesh N, Steele-Stallard HB, Ambrose J, Coffey A Emmerson J, Haralambous E, Hughes Y, Steel KP, et al. Comprehensive sequence analysis of nine usher syndrome genes in the UK National Collaborative Usher Study. J Med Genet. 2012;49(1):27-36.

12. Nagase $Y$, Kurata K, Hosono K, Suto K, Hikoya A, Nakanishi H, Mizuta K, Mineta H, Minoshima S, Hotta Y. Visual outcomes in Japanese patients with retinitis Pigmentosa and usher syndrome caused by USH2A mutations. Semin Ophthalmol. 2018;33(4):560-5.

13. Jacobson SG, Cideciyan AV, Aleman TS, Sumaroka A, Roman AJ, Gardner LM, Prosser HM, Mishra M, Bech-Hansen NT, Herrera W, et al. Usher syndromes due to MYO7A, PCDH15, USH2A or GPR98 mutations share retinal disease mechanism. Hum Mol Genet. 2008:17(15):2405-15.

14. Mathur PD, Zou J, Zheng T, Almishaal A, Wang Y, Chen Q, Wang L, Vashist D, Brown S, Park A, et al. Distinct expression and function of whirlin isoforms in the inner ear and retina: an insight into pathogenesis of USH2D and DFNB31. Hum Mol Genet. 2015:24(21):6213-28.

15. Ebermann I, Phillips JB, Liebau MC, Koenekoop RK, Schermer B, Lopez I, Schafer E, Roux AF, Dafinger C, Bernd A, et al. PDZD7 is a modifier of retinal disease and a contributor to digenic usher syndrome. J Clin Invest. 2010; 120(6):1812-23.

16. Hartel BP, van Nierop JWI, Huinck WJ, Rotteveel LJC, Mylanus EAM, Snik AF, Kunst HPM, Pennings RJE. Cochlear implantation in patients with usher syndrome type lla increases performance and quality of life. Otol Neurotol. 2017:38(6):e120-7

17. McMillan DR, Kayes-Wandover KM, Richardson JA, White PC. Very large G protein-coupled receptor-1, the largest known cell surface protein, is highly expressed in the developing central nervous system. J Biol Chem. 2002; 277(1):785-92.

18. Gerber S, Bonneau D, Gilbert B, Munnich A, Dufier JL, Rozet JM, Kaplan J. USH1A: chronicle of a slow death. Am J Hum Genet. 2006;78(2):357-9.

19. Petit C. Usher syndrome: from genetics to pathogenesis. Annu Rev Genomics Hum Genet. 2001;2:271-97.

20. Reiners J, Nagel-Wolfrum K, Jurgens K, Marker T, Wolfrum U. Molecular basis of human usher syndrome: deciphering the meshes of the usher protein network provides insights into the pathomechanisms of the usher disease. Exp Eye Res. 2006;83(1):97-119.

21. Arno G, Agrawal SA, Eblimit A, Bellingham J, Xu M, Wang F, Chakarova C, Parfitt DA, Lane A, Burgoyne T, et al. Mutations in REEP6 cause autosomalrecessive retinitis Pigmentosa. Am J Hum Genet. 2016;99(6):1305-15.

22. Wang F, Wang H, Tuan HF, Nguyen DH, Sun V, Keser V, Bowne SJ, Sullivan LS, Luo $H$, Zhao L, et al. Next generation sequencing-based molecular diagnosis of retinitis pigmentosa: identification of a novel genotype-phenotype correlation and clinical refinements. Hum Genet. 2014;133(3):331-45

23. Pollak A, Lechowicz U, Murcia Pienkowski VA, Stawinski P, Kosinska J, Skarzynski H, Oldak M, Ploski R. Whole exome sequencing identifies TRIOBP pathogenic variants as a cause of post-lingual bilateral moderate-to-severe sensorineural hearing loss. BMC medical genetics. 2017;18(1):142.

24. $\mathrm{Fu} \mathrm{J,} \mathrm{Li} \mathrm{L,} \mathrm{Lu} \mathrm{G.} \mathrm{Relationship} \mathrm{between} \mathrm{microdeletion} \mathrm{on} \mathrm{Y} \mathrm{chromosome} \mathrm{and}$ patients with idiopathic azoospermia and severe oligozoospermia in the Chinese Chin Med J. 2002;115(1):72-5.
25. Fu S, Cheng J, Wei C, Yang L, Xiao X, Zhang D, Stewart MD, Fu J. Development of diagnostic SCAR markers for genomic DNA amplifications in breast carcinoma by DNA cloning of high-GC RAMP-PCR fragments. Oncotarget. 2017:8(27):43866-77.

26. Zhu L, Cheng J, Zhou B, Wei C, Yang W, Jiang D, ljaz I, Tan X, Chen R, Fu J. Diagnosis for choroideremia in a large Chinese pedigree by nextgeneration sequencing (NGS) and noninvasive prenatal testing (NIPT). Mol Med Rep. 2017;15(3):1157-64.

27. Yuan Z, Li B, Xu M, Chang EY, Li H, Yang L, Wu S, Soens ZT, Li Y, Wong LC, et al. The phenotypic variability of HK1-associated retinal dystrophy. Sci Rep. 2017;7(1):7051.

28. Ge Z, Bowles K, Goetz K, Scholl HP, Wang F, Wang X, Xu S, Wang K, Wang $\mathrm{H}$, Chen R. NGS-based molecular diagnosis of 105 eyeGENE((R)) probands with retinitis Pigmentosa. Sci Rep. 2015;5:18287.

29. Li H, Durbin R. Fast and accurate short read alignment with burrowswheeler transform. Bioinformatics. 2009;25(14):1754-60.

30. Marchler-Bauer A, Bo Y, Han L, He J, Lanczycki CJ, Lu S, Chitsaz F, Derbyshire MK, Geer RC, Gonzales NR, et al. CDD/SPARCLE: functional classification of proteins via subfamily domain architectures. Nucleic Acids Res. 2017;45(D1):D200-3.

31. Fu J, Qin L, He T, Qin J, Hong J, Wong J, Liao L, Xu J. The TWIST/Mi2/NuRD protein complex and its essential role in cancer metastasis. Cell Res. 2011; 21(2):275-89.

32. Fu J, Zhang L, He T, Xiao X, Liu X, Wang L, Yang L, Yang M, Zhang T, Chen R, et al. TWIST represses estrogen receptor-alpha expression by recruiting the NuRD protein complex in breast cancer cells. Int J Biol Sci. 2012;8(4):522-32.

33. Friedman TB, Schultz JM, Ahmed ZM, Tsilou ET, Brewer CC. Usher syndrome: hearing loss with vision loss. Adv Otorhinolaryngol. 2011;70:56-65.

34. Weston MD, Luijendijk MW, Humphrey KD, Moller C, Kimberling WJ. Mutations in the VLGR1 gene implicate G-protein signaling in the pathogenesis of usher syndrome type II. Am J Hum Genet. 2004;74(2):357-66.

35. Imani S, Cheng J, Shasaltaneh MD, Wei C, Yang L, Fu S, Zou H, Khan MA, Zhang $X$, Chen $\mathrm{H}$, et al. Genetic identification and molecular modeling characterization reveal a novel PROM1 mutation in Stargardt4-like macular dystrophy. Oncotarget. 2018;9(1):122-41.

36. Imani S, Cheng J, Mobasher-Jannat A, Wei C, Fu S, Yang L, Jadidi K, Khosravi $\mathrm{MH}$, Mohazzab-Torabi S, Shasaltaneh MD, et al. Identification of a novel RPGRIP1 mutation in an Iranian family with leber congenital amaurosis by exome sequencing. J Cell Mol Med. 2018;22(3):1733-42.

37. Schwartz SB, Aleman TS, Cideciyan AV, Windsor EA, Sumaroka A, Roman AJ, Rane T, Smilko EE, Bennett J, Stone EM, et al. Disease expression in usher syndrome caused by VLGR1 gene mutation (USH2C) and comparison with USH2A phenotype. Invest Ophthalmol Vis Sci. 2005;46(2):734-43.

38. Michalski N, Michel V, Bahloul A, Lefevre G, Barral J, Yagi H, Chardenoux S, Weil D, Martin P, Hardelin JP, et al. Molecular characterization of the anklelink complex in cochlear hair cells and its role in the hair bundle functioning. J Neurosci. 2007;27(24):6478-88.

39. Zou J, Zheng T, Ren C, Askew C, Liu XP, Pan B, Holt JR, Wang Y, Yang J. Deletion of PDZD7 disrupts the usher syndrome type 2 protein complex in cochlear hair cells and causes hearing loss in mice. Hum Mol Genet. 2014; 23(9):2374-90.

40. Hmani-Aifa M, Benzina Z, Zulfiqar F, Dhouib H, Shahzadi A, Ghorbel A, Rebai A, Soderkvist P, Riazuddin S, Kimberling WJ, et al. Identification of two new mutations in the GPR98 and the PDE6B genes segregating in a Tunisian family. Eur J Hum Genet. 2009;17(4):474-82.

41. Ebermann I, Wiesen MH, Zrenner E, Lopez I, Pigeon R, Kohl S, Lowenheim H, Koenekoop RK, Bolz HJ. GPR98 mutations cause usher syndrome type 2 in males. J Med Genet. 2009:46(4):277-80.

42. McGee J, Goodyear RJ, McMillan DR, Stauffer EA, Holt JR, Locke KG, Birch DG, Legan PK, White PC, Walsh EJ, et al. The very large G-protein-coupled receptor VLGR1: a component of the ankle link complex required for the normal development of auditory hair bundles. J Neurosci. 2006;26(24):6543-53.

43. Chen Q, Zou J, Shen Z, Zhang W, Yang J. Whirlin and PDZ domaincontaining 7 (PDZD7) proteins are both required to form the quaternary protein complex associated with usher syndrome type 2. J Biol Chem. 2014; 289(52):36070-88

44. Imani S, ljaz I, Shasaltaneh MD, Fu S, Cheng J, Fu J. Molecular genetics characterization and homology modeling of the CHM gene mutation: a study on its association with choroideremia. Mutat Res. 2018;775:39-50. 